\title{
Silicon Nanomembranes as a Means to Evaluate Stress Evolution in Deposited Thin Films
}

Anna M. Clausen ${ }^{\mathrm{a}, 1, \#}$, Deborah M. Paskiewicz ${ }^{\mathrm{a}, 2, \#}$, Alireza Sadeghirad ${ }^{\mathrm{b}, 3}$, Joseph Jakes $^{\mathrm{c}}$, Donald E. Savage $^{\mathrm{a}}$, Donald S. Stone ${ }^{\mathrm{a}}$, Feng Liu ${ }^{\mathrm{b}}$, and Max G. Lagally ${ }^{\mathrm{a}, *}$

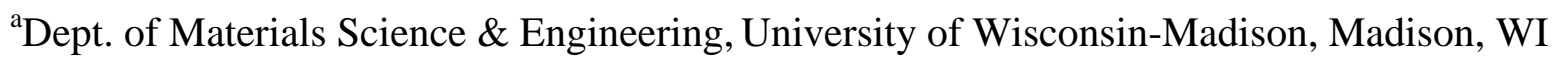
53706

${ }^{\mathrm{b}}$ Dept. of Materials Science \& Engineering, University of Utah, Salt Lake City, UT 84112

${ }^{c}$ Forest Biopolymers Science and Engineering, USDA Forest Service, Forest Products Laboratory, Madison, WI 53726

*Corresponding Author Email: lagally@engr.wisc.edu

\#These authors contributed equally to this work.

\section{$\underline{\text { Abstract }}$}

Thin-film deposition on ultra-thin substrates poses unique challenges because of the potential for a dynamic response to the film stress during deposition. While theoretical studies have investigated film stress related changes in the substrate, little has been done to learn how stress might evolve in a film growing on a compliant substrate. We use silicon nanomembranes (SiNMs), extremely thin sheets of single-crystalline $\mathrm{Si}$, as a substrate for the growth of amorphous $\mathrm{SiN}_{\mathrm{x}}$ to begin to address this question. Nanomembranes are released from a silicon-oninsulator wafer with selective etching, transferred over a hole etched into a Si wafer, and bonded to the edges of the hole. The nanomembrane window provides the substrate for $\mathrm{SiN}_{\mathrm{x}}$ deposition and a platform, using Raman spectroscopy, for measurements of the evolving strain in the nanomembrane. From the strain in the nanomembrane, the film stress can be inferred from the required balance of forces in the film/substrate system. We observe that the strain in the tethered

\footnotetext{
${ }^{1}$ Present address: Intel Corporation, Chandler, AZ 85248

${ }^{2}$ Present address: Argonne National Laboratory, Argonne, IL 60439

${ }^{3}$ Present address: Global Engineering and Materials, Princeton, NJ 08540

(C) 2014. This manuscript version is made available under the Elsevier user license http://www.elsevier.com/open-access/userlicense/1.0/
} 
NM increases as the NM is made thinner while the intrinsic steady-state stress in the deposited film is reduced.

Keywords: Thin Films; Silicon; Nanomaterials; Stress and Strain; Nanomembranes

\section{Introduction}

Thin-film deposition on thick substrates is widely used and has been extensively investigated. The stress that forms in these systems can be characterized and quantified by classical analysis $[1,2]$ of the physical effect the film has on the substrate (e.g., bending, cracking, etc.). In modern applications, substrates may, however, be quite thin. One may expect that the stress in a thin film deposited on a thin substrate will evolve differently from that of a similar film deposited on a thick substrate, because in such systems the strain can be shared during deposition [3]. Silicon nanomembranes (NMs), thin sheets of single-crystalline Si, potentially provide the platform to investigate such stress evolution [4], because the Raman spectrum in Si is very sensitive to strain $[5,6]$. Eventually one would hope to use a thin crystalline Si sheet as a strain gauge to evaluate the stress in a film at various stages in the deposition, from that determine if the stress in the film builds up differently if it is grown on a thick or a thin substrate, and thereby begin to understand better stress evolution during the growth of thin films.

Semiconductor nanomembranes have evolved in the last decade into a major platform for both fundamental studies and novel device applications [7-10], with a major driving force being the application of or the response to strain. In the pursuit of new physical properties, Group IV NMs have been strained in a controlled manner, among others, to modify the band structure or band offsets $[11,12]$, to change the strain symmetry [13], or to create improved two-dimensional electron gases $[14,15]$. In the pursuit of nanoarchitectures or new devices, Group IV NMs have 
been rolled into tubes [10, 16-18] or supported channels [19]; or bonded to flexible supports [7, 20-24] or curved surfaces [25] for electronic- and optoelectronic-device applications.

For growth on thin substrates, strain sharing $[3,4]$ has been observed for the epitaxy of nanocrystals on SiNMs [26-28]. In these systems, the strain transfer was analyzed by modeling the local and global bending of the NM. Here we investigate deposition of an amorphous, presumably continuous, compressive silicon nitride $\left(\mathrm{SiN}_{\mathrm{x}}\right)$ film, and use Raman spectroscopy as the major tool to analyze strain, and thus stress in the deposited film.

\subsection{Stress-strain relationships in thin films}

In conventional thin-film deposition on thick, rigid substrates (thickness of substrate, $\mathrm{h}_{\mathrm{s}}$ $>>$ thickness of film, $\mathrm{h}_{\mathrm{f}}$ ), as long as the film adheres to the substrate, the stress in the film is inferred from the resulting curvature of the substrate. Stoney's equation [1] relates the curvature of the substrate, $\kappa$, to the physical properties of the substrate (biaxial modulus, $\mathrm{M}_{\mathrm{s}}$, and thickness, $h_{s}$ ), the thickness of the film, $h_{f}$, and the stress in the film, $\sigma_{\mathrm{f}}$ :

$$
=\frac{6{ }_{f} h_{f}}{M_{s} h_{s}^{2}}
$$

Because it is assumed that the substrate is much thicker than the film $\left(h_{s}>>h_{f}\right)$, effectively all the elastic strain in the system is in the film. The elastic strain in the film, $\varepsilon_{\mathrm{f}}$, is related to the film stress by the biaxial modulus of the film $\left(\varepsilon_{\mathrm{f}}=\sigma_{\mathrm{f}} / \mathrm{M}_{\mathrm{f}}\right)$. The strain in the system is defined as the difference between the strain in the film and the strain in the substrate, $\varepsilon_{\mathrm{m}} \equiv \varepsilon_{\mathrm{f}}-\varepsilon_{\mathrm{s}}$. Therefore, when $\mathrm{h}_{\mathrm{s}}>>\mathrm{h}_{\mathrm{f}}, \varepsilon_{\mathrm{m}}=\varepsilon_{\mathrm{f}}$.

If the substrate thickness is reduced such that $h_{s}$ is no longer much larger than $h_{f}$, the total strain in the system is shared between the film and the substrate. The system as a whole will expand or contract (and curl) according to the system strain $[3,10]$. To illustrate this concept, consider a thin film with some strain, $\varepsilon_{\mathrm{f}}$, grown on a bulk substrate such that initially $\varepsilon_{\mathrm{m}}=\varepsilon_{\mathrm{f}}$. Figure 
1 shows a film grown on both sides of the substrate so that curvature in the system can be ignored: bending moments from either side of the substrate cancel each other such that only expansion or compression of the system needs to be considered. If one then imagines that the substrate is thinned (even though that is not experimentally possible with a film on both sides), some of the system strain, $\varepsilon_{\mathrm{m}}$, is transferred to the substrate. Now the system strain is shared between the film layers and the substrate $\left(\varepsilon_{\mathrm{m}}=\varepsilon_{\mathrm{f}}-\varepsilon_{\mathrm{s}}\right)$. Using this strain sharing condition and a balance of forces between the layers,

$$
\begin{aligned}
{ }_{s} h_{s} & ={ }_{f} h_{f} \\
M_{s s} h_{s} & =M_{f f} h_{f}
\end{aligned}
$$

one can predict the strain in the substrate or the film based on the thicknesses and the biaxial moduli of the layers [2]:

$$
\begin{aligned}
& { }_{s}=\frac{M_{f} h_{f}}{M_{f} h_{f}+M_{s} h_{s}}{ }_{m} \\
& { }_{f}=\frac{M_{s} h_{s}}{M_{f} h_{f}+M_{s} h_{s}}{ }_{m} .
\end{aligned}
$$

Equations 3 describe the strain in heterostructures for which there is a well defined system strain. An illustration of the strain in the film and the substrate as a function of substrate thickness (based on Equations 3) is shown in Figure 1c. As the substrate is thinned, more of the system strain is transferred to the substrate. When the substrate is much thicker than the film, the substrate strain approaches zero and the film strain approaches the system strain, as was initially defined above. The thickness at which the film and substrate strain are equal will depend on the elastic properties of the film and substrate $\left(\mathrm{M}_{\mathrm{f}}, \mathrm{M}_{\mathrm{s}}\right)$ and the thickness of the film $\left(\mathrm{h}_{\mathrm{f}}\right)$ [for fixed substrate thickness]. 


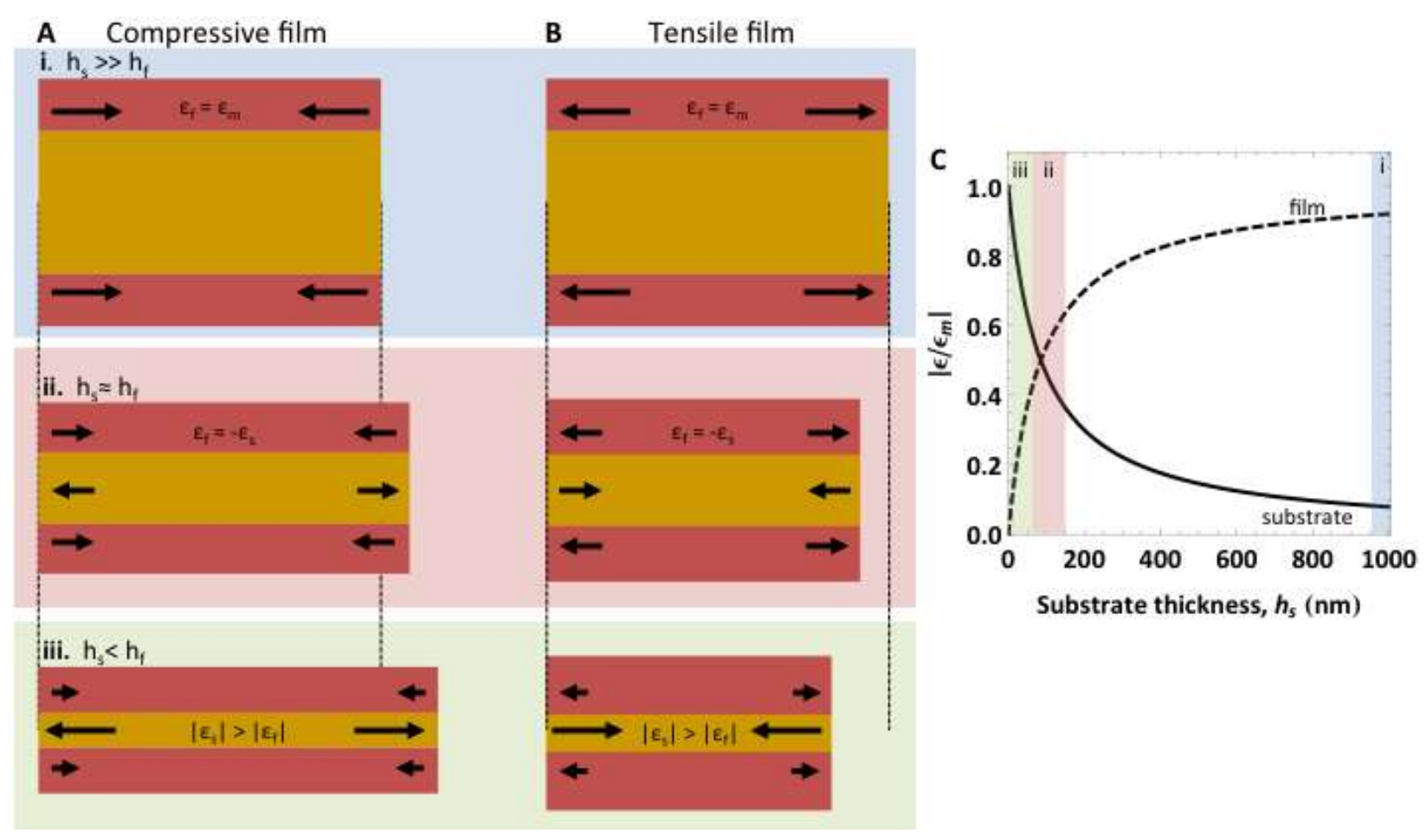

Figure 1. Illustration of strain sharing between a film and substrate as the substrate is thinned. Initially, all the strain in the system is in the film (i). As the substrate is thinned, some of the strain is transferred to the substrate (ii). Once the substrate is thinner than the total film thickness the strain magnitude in the substrate exceeds that in the film (iii). (A) Compressive strain in the film is transferred as tensile strain to the substrate. (B) Tensile strain in the film is transferred as compressive strain to the substrate. The schematic diagrams in (A) and (B) show a film deposited on both sides of the substrate such that the bending moment can be neglected. (C) Plot of the normalized strain magnitude. The elastic properties of film and substrate are assumed equal $\left(\mathrm{M}_{\mathrm{f}}=\mathrm{M}_{\mathrm{s}}\right)$ and that the total film thickness (sum of top and bottom films), $\mathrm{h}_{\mathrm{f}}$, is constant at $100 \mathrm{~nm}$.

\subsection{Stress evolution in non-epitaxial thin films on ultrathin substrates}

The strain sharing approach described above will be able to predict the strain evolution in both the film and substrate during film growth on a thin substrate only if the system strain $\left(\varepsilon_{\mathrm{m}}\right)$ is well defined, as would be the case for coherent epitaxial growth. For the general growth case 
(non-epitaxial polycrystalline or amorphous, on a thin crystalline substrate), we can only infer the system strain from the strain measured in the thin crystalline substrate after deposition of some quantity of material. We will thus use stress when referring to non-epitaxial films. For simplicity, we will continue to use strain when referring to single-crystalline films and substrates.

For growth on an ultrathin substrate, instead of the substrate remaining static during film deposition, it is free to expand or contract in response to the stress generated in the depositing film. For a film that has a tensile stress, the substrate will contract during deposition to balance the forces in the system. Conversely, for a film with a compressive stress, the substrate will expand during deposition. The thin substrate is continuously reacting to the stress in the film at any given moment during deposition by expanding or contracting based on the thickness of the film relative to the thickness of the substrate and the stress evolution in the film. The instantaneous strain in the thin substrate will be a result of the balance of forces between the stresses in the film and in the substrate (Eq. (2) rearranged):

$$
{ }_{s}=\frac{f_{f}}{M_{s} h_{s}} .
$$

We can determine how the stress in a film deposited on a compliant substrate behaves by changing either the film or the substrate thickness and measuring the resulting substrate strain at each condition. The substrate strain, a measurable quantity, will help us determine if the stress in the film is a function of the deposited-film thickness, and if the magnitude of stress in the film is different from the stress in films deposited on rigid substrates under the same conditions and to the same thicknesses.

\section{Experimental}

In order to evaluate whether a compliant substrate can eventually be exploited to determine in real time the evolving film stress in a growing film (possibly even with lateral spatial 
resolution), we perform the "static" analogue. As our substrates we use Si NMs that are transferred to a window cut into a thick Si wafer and bonded on all sides to this wafer, in analogy to a window frame, as shown in Fig. 2. Using Raman spectroscopy, we measure the strain in the freestanding portion of the Si NMs [4], far from the bonded edges. We then systematically vary either the thickness of these Si NM substrates or the deposited-film thickness, measure the shift of the relevant Raman peak in the substrate, and infer the stress in the deposited film. We investigate the stress in deposited amorphous silicon nitride $\left(\operatorname{SiN}_{x}\right) . \operatorname{SiN}_{x}$ is chosen because one can deposit a composition that is always compressive, necessary for this experiment, as detailed below.

The bonded-window-frame is not ideal, as it prevents the Si NM substrate from responding to stress as a completely free-standing NM would. The tethered edges prevent the NM substrate from contracting if a deposited film were to create compressive strain in the NM; however, the tethered edges are needed to prevent the film/NM bilayer system from curling [18]. A better approach would be a vertically suspended cantilever, with deposition on both sides, but this was not possible with our deposition method. Given these constraints, we chose a film material $\left(\mathrm{SiN}_{\mathrm{x}}\right)$ that is known to be under compression at specified deposition conditions, such that the overall system strain is compressive and the substrate will expand (and thus be in tension) as the deposited film attempts to relieve stress.

The Si NMs start as the template layer of silicon-on-insulator (SOI) wafers. Fig. 2 shows a combination of schematic diagrams and optical-microscopy images of the procedure for making and using the NM window. Details are provided in Methods. 

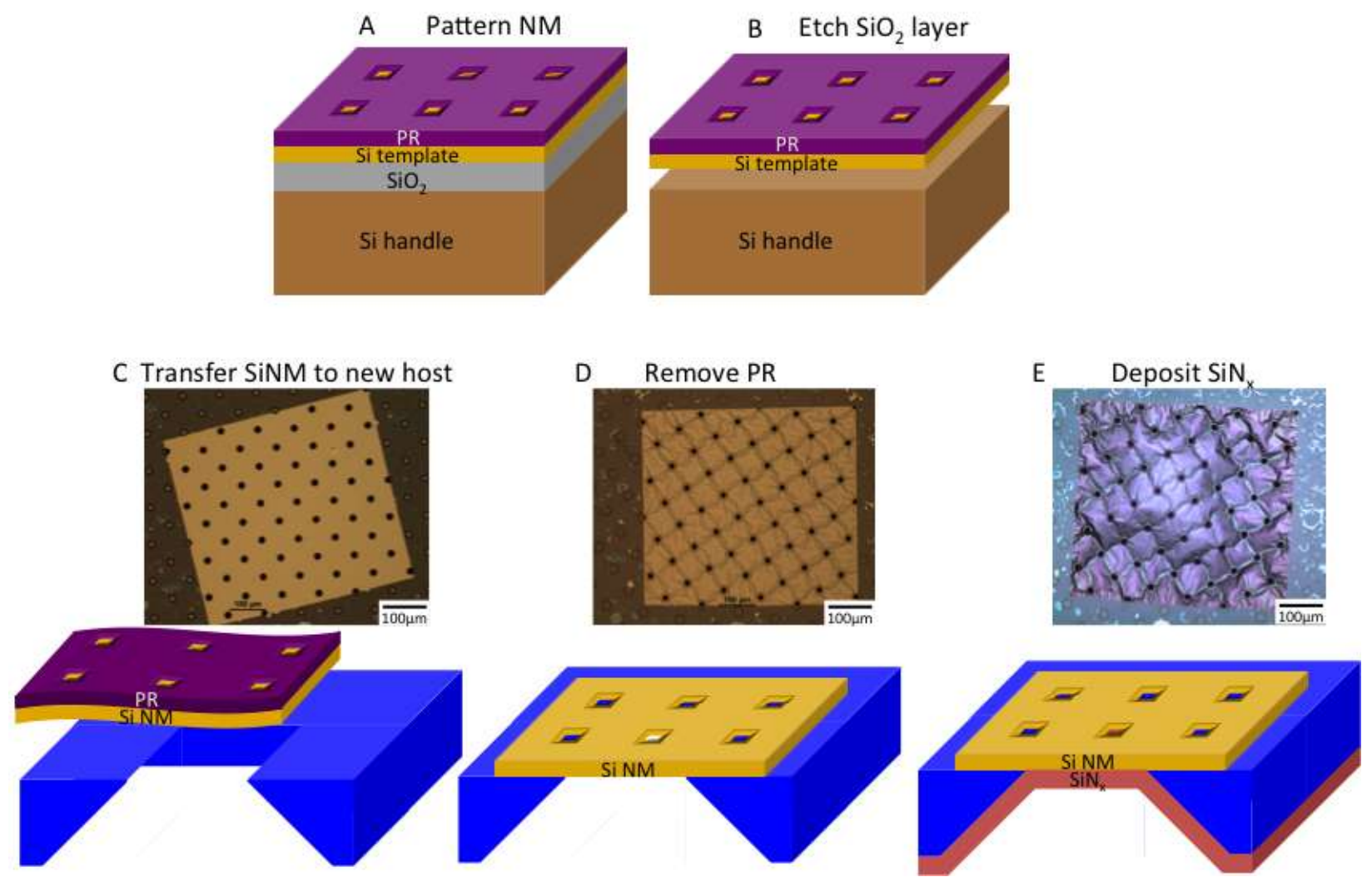

Figure 2. Fabrication and use of tethered Si NMs. (A) Preparation of the Si template layer of SOI. (B) $\mathrm{The}^{\mathrm{SiO}_{2}}$ layer is selectively etched with HF to release the Si template. (C) Transfer of the Si NM over a hole etched into a Si wafer. The photoresist (PR) remains on the Si template layer during transfer of the Si NM. The optical micrograph shows a flat NM. (D) Removal of the PR. The optical micrograph shows small wrinkles, indicative of the release of a small amount of compressive strain. (E) $\mathrm{SiN}_{\mathrm{x}}$ deposition on one side of the tethered Si NM. The Si NM has expanded again, as indicated by the increase in the deformation of the tethered Si NM in the optical micrograph.

Once the tethered $\mathrm{Si}$ NM substrate is prepared, amorphous $\mathrm{SiN}_{\mathrm{x}}$ is deposited, with plasma enhanced chemical vapor deposition (PECVD), onto the backside of the Si NM (through the hole in the $\mathrm{Si}$ wafer) [Figure $2 \mathrm{E}$ ]. We keep the recipe constant for all $\mathrm{SiN}_{\mathrm{x}}$ depositions and only vary the deposition time to alter the film thickness.

The top surface of the ultrathin Si NM is used for strain and morphological characterization. We used white-light interferometry to measure the deformation, and Raman spectroscopy to 
measure the strain in the Si NMs. The Raman active modes of Si shift linearly with strain [5]. We assume that the strain in the $\mathrm{Si}$ is equibiaixal, given that the $\mathrm{Si}$ is (001)-oriented and the $\mathrm{SiN}_{\mathrm{x}}$ stressor film is amorphous [13]. Raman spectra are acquired at several points on the freestanding region (away from the etchant access holes) of the sample before and after $\mathrm{SiN}_{\mathrm{x}}$ deposition. We average the frequency shifts from all similar measurements to calculate an average strain in the ultrathin Si NM substrate [6].

We examine the stress in the amorphous $\mathrm{SiN}_{\mathrm{x}}$ films deposited on the SiNM with two experiments: (1) we vary the $\mathrm{SiN}_{\mathrm{x}}$ film thickness (90-150nm) and keep the NM (substrate) thickness constant (53nm), and (2) we vary the thickness of the NM substrate (9-200nm) while keeping the thickness of the $\mathrm{SiN}_{\mathrm{x}}$ film constant (100nm). The deformation and strain in the Si NM substrates is evaluated before and after $\operatorname{SiN}_{\mathrm{x}}$ deposition to separate any possible effects caused by the transfer of the NMs to the $\mathrm{Si}$ windows from the changes caused by the $\mathrm{SiN}_{\mathrm{x}}$ deposition. Only the strain resulting from the $\operatorname{SiN}_{\mathrm{x}}$ deposition (final strain minus initial strain) is used to model the strain in the Si NM substrates as a function of film or substrate thickness.

The Young's modulus of $\mathrm{SiN}_{\mathrm{x}}$ films deposited on bulk substrates was determined by nanoindentation experiments. $\mathrm{SiN}_{\mathrm{x}}$ films with thicknesses ranging from 60-182nm were deposited on bulk (100) Si wafers. A value of $E=120 \pm 20 \mathrm{GPa}$ is estimated for $\mathrm{SiN}_{\mathrm{x}}$. To determine the biaxial modulus $[\mathrm{M}=\mathrm{E} /(1-v)]$, we assumed that the Poisson ratio $(v)$ of the $\mathrm{SiN}_{\mathrm{x}}$ film is similar to stoichiometric $\mathrm{Si}_{3} \mathrm{~N}_{4}(v=0.25$ [2]). More details can be found in Methods.

\section{Results and Discussion}

We expect that the steady-state stress in the deposited $\mathrm{SiN}_{\mathrm{x}}$ films should be independent of film thickness. To show that this is true, we deposited uniform $\mathrm{SiN}_{\mathrm{x}}$ films of varying thick- 
nesses on bulk Si wafers and measured the curvature of the wafer as a function of film thickness. A linear relationship between wafer curvature and film thickness, shown in Fig. 3, demonstrates that the stress in the $\mathrm{SiN}_{\mathrm{x}}$ is not a function of film thickness $\left(\sigma_{\mathrm{f}}=-192 \mathrm{MPa}\right)$, as predicted by Stoney's equation for stressed thin films on thick substrates [1].

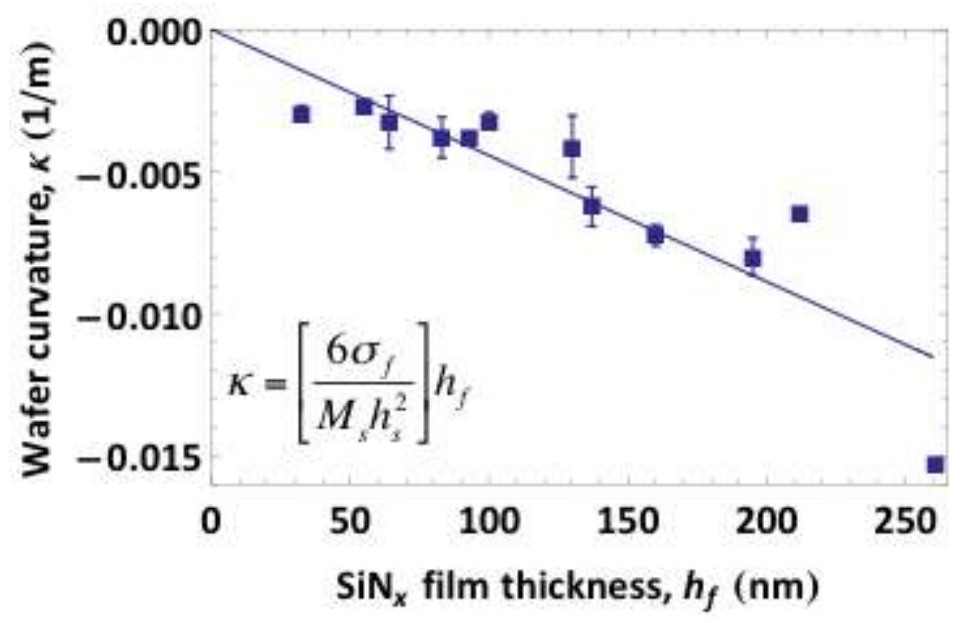

Figure 3. Plot of the wafer curvature, $\kappa$, as a function of the thickness $\left(\mathrm{h}_{\mathrm{f}}\right)$ of $\mathrm{SiN}_{\mathrm{x}}$ deposited on 3-inch bulk Si substrates $\left(\mathrm{h}_{\mathrm{s}}=375 \mu \mathrm{m}, \mathrm{M}_{\mathrm{s}}=180 \mathrm{GPa}\right)$. From Stoney's equation (Eq. 1), the slope of the best-fit line is proportional to the biaxial stress in the $\operatorname{SiN}_{\mathrm{x}}$ film $\left(\sigma_{\mathrm{f}}=-192 \mathrm{MPa}\right)$.

In the first set of depositions we prepared, the Si NM thickness is held constant (53nm) and the $\mathrm{SiN}_{\mathrm{x}}$ thickness is varied from $90-150 \mathrm{~nm}$ to investigate any possible thickness dependence of the $\mathrm{SiN}_{\mathrm{x}}$ film stress when deposited on extremely thin substrates. This experiment is the analogue of that shown in Fig. 3, i.e., depositing varying-thickness $\mathrm{SiN}_{\mathrm{x}}$ films on bulk to determine the stress in the deposited film. Now, however, the strain in the Si NMs is extracted from Raman measurements; it is plotted as a function of $\mathrm{SiN}_{\mathrm{x}}$ thickness in Figure 4A. The linear dependence of the Si NM strain as a function of $\mathrm{SiN}_{\mathrm{x}}$ thickness indicates that the stress in the deposited film is again constant, to the accuracy to which we can measure it. A best fit is shown by 
the solid line in Figure 4A; it gives a film stress, $\sigma_{\mathrm{f}}=-121 \pm 12 \mathrm{MPa}$, a value lower than the $\operatorname{SiN}_{\mathrm{x}}$ film stress extracted from films deposited on bulk substrates $\left(\sigma_{\mathrm{f}}=-192 \mathrm{MPa}\right.$, Fig.3 $)$.

In Figure 4A, we also plot how the strain in the Si NM substrate should change with $\mathrm{SiN}_{\mathrm{x}}$ thickness (red dashed line) if there was a constant system $\underline{\operatorname{strain}}\left(\varepsilon_{\mathrm{m}}=\varepsilon_{\mathrm{f}}-\varepsilon_{\mathrm{s}}\right)$ as modeled in Figure 1 . The system strain in the model plotted in Fig 1 is equal to the equivalent strain in similar $\operatorname{SiN}_{\mathrm{x}}$ films deposited on bulk substrates $\left(\varepsilon_{\mathrm{m}}=\varepsilon_{\mathrm{f}}, \varepsilon_{\mathrm{f}}=\sigma_{\mathrm{f}} / \mathrm{M}_{\mathrm{f}}=-192 \mathrm{MPa} / 154 \mathrm{GPa}=-0.125 \%\right) . \operatorname{Re}-$ call that this model assumes that the films deposited on bulk substrates have the same stress evolution as those deposited on ultrathin substrates; there is no dynamic reaction of the substrate during deposition. Clearly this model does not describe the experimentally observed relationship between the strain in the Si NM substrate and the $\mathrm{SiN}_{\mathrm{x}}$ film thickness. Therefore, the nanomembrane substrates must be dynamically changing during the film deposition, thus altering the strain in the deposited film, but surprisingly in such a manner as to keep the deposited-film stress constant.

We investigated the strain in the Si NM substrates in the complementary experiment: constant $\mathrm{SiN}_{\mathrm{x}}$ film thickness (100nm) and varying Si NM substrate thickness (9-200nm). The results are shown in Fig. 4B. The models plotted in Figure 4A are also plotted in Figure 4B: (1) constant film stress as measured on 53nm Si NM substrates $\left(\sigma_{\mathrm{f}}=-121 \mathrm{MPa}\right.$, solid black line $),(2)$ constant film stress as measured on bulk substrates $\left(\sigma_{\mathrm{f}}=-192 \mathrm{MPa}\right.$, dashed black line), and (3) constant system strain based on Eqn. $3 b\left(\varepsilon_{\mathrm{f}}-\varepsilon_{\mathrm{s}}=\varepsilon_{\mathrm{m}}=-0.125 \%\right.$, dashed red line $)$. The curves for the two values of stress bracket the data; because of the uncertainties in the measurements, it is difficult to be more reliably specific. However, the measured strain in the substrate appears to be closer to the lower stress value $\left(\sigma_{\mathrm{f}}=-121 \mathrm{MPa}\right.$ solid line $)$ for thinner substrates and closer to the bulk stress value $\left(\sigma_{\mathrm{f}}=-192 \mathrm{MPa}\right.$ dashed line $)$ as the substrate thickness increases. The constant 
system strain model (red line) clearly does not fit. As the substrate thickness approaches 200nm, all three models converge within experimental uncertainty. This result suggests that at these thicknesses the NM substrate no longer reacts noticeably during film deposition; the substrate appears bulk-like to the film and the film stress evolves as it would when grown on a noncompliant substrate.
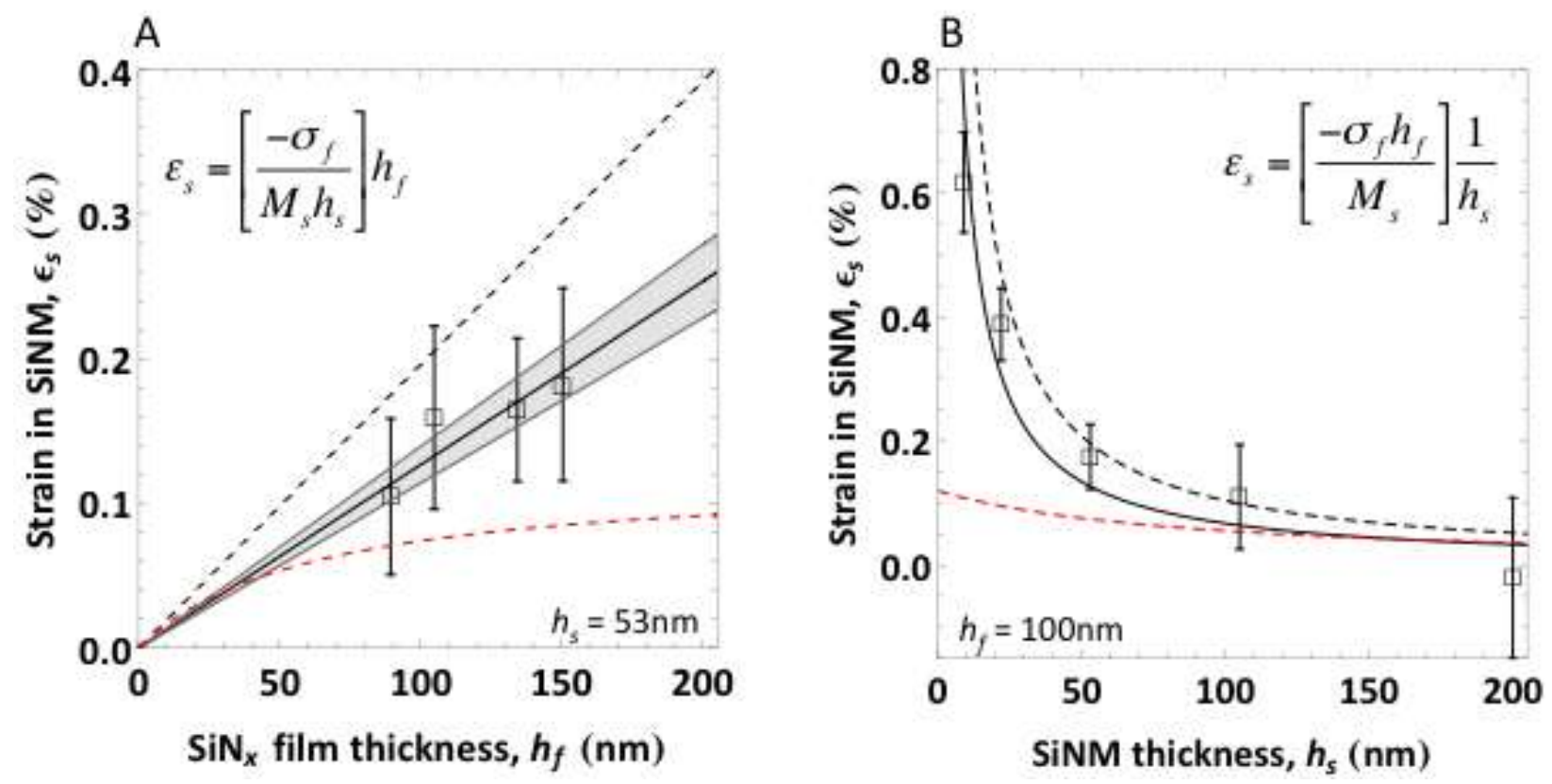

Figure 4. (A) Average strain in the $\mathrm{Si} \mathrm{NM}$ substrates as a function of $\mathrm{SiN}_{\mathrm{x}}$ film thickness and (B) Si NM substrate thickness. The data represent the strain in the Si NM substrates from the $\mathrm{SiN}_{\mathrm{x}}$ deposition only. In (A), a constant film stress of $-121 \pm 12 \mathrm{MPa}$ is extracted from the linear relationship between the strain in the Si NM substrate and the $\mathrm{SiN}_{\mathrm{x}}$ film thickness (solid black line). The gray shaded region in (A) denotes the uncertainty in the stress in films deposited on 53nm Si NM substrates. The dashed black line denotes the constant film stress extracted from films deposited on bulk substrates $\left(\sigma_{\mathrm{f}}=-192 \mathrm{MPa}\right)$. The dashed red line is a model for the strain in the Si NM substrate assuming a constant system strain.

The models described above account only for stretching of the ultrathin Si NM sub-

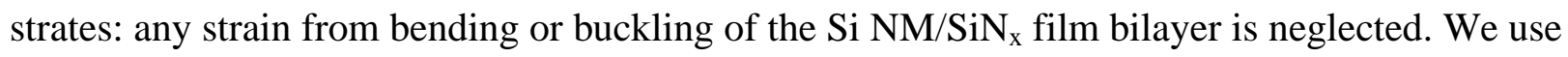


finite element analysis (FEA) to model the $\mathrm{Si} \mathrm{NM} / \mathrm{SiN}_{\mathrm{x}}$ bilayer system with constrained edges. We examine the strain and buckling distribution over the freestanding region of our samples to test if the above assumption is valid. A constant system strain of $-0.27 \%$ (equivalent to the measured system strain for a $125 \mathrm{~nm} \mathrm{SiN}_{\mathrm{x}}$ film deposited on a $22 \mathrm{~nm} \mathrm{Si} \mathrm{NM}$ ) is applied to the bilayer to examine the distribution of strain in, and the magnitude of the deflection of, the tethered Si NM substrate after $\mathrm{SiN}_{\mathrm{x}}$ deposition. It is important to note that the system strain is the inferred strain difference between the Si NM and the $\operatorname{SiN}_{x}$ film ( $\varepsilon_{m}$ as calculated with Equation 3a). The FEA model does not describe the stress evolution in thin films deposited on the thin substrates, only the resulting strain distribution and buckling (deflection) based on the above assumptions.

Figure 5 shows the strain distribution for the same sample parameters listed above. In the FEA model, we also assumed that the initial strain in the Si NM substrate was zero, such that the final strain is equivalent to the strain imposed on the substrate by only the deposited film (system strain $\left.=\varepsilon_{\mathrm{m}}=-0.27 \%\right)$. Any strain from the fabrication of the tethered Si NM substrates is neglected. The magnitude of the strain in the results is equivalent to the difference in strain measured before and after film deposition. Figure 5 shows that the distribution of strain in the Si NM after film deposition is fairly uniform. Any strain inhomogeneities that are observed in Fig. 5 are a result of the constrained edges and the free surfaces created by the etchant access holes in the NM. The magnitude of strain in the NM from strain sharing with the deposited film will be much larger than any strain from buckling of the NM due to its expansion from strain sharing.

Our measurements were taken in areas between etchant access holes and away from the tethered edges of the Si NM: in other words, in areas where the strain appears most uniform. We thus believe that our Raman measurements fairly represent the NM strain. The FEA model pro- 
duces an average strain in the Si NM of $0.22 \%$, which matches the $0.23 \pm 0.02 \%$ strain measured experimentally.
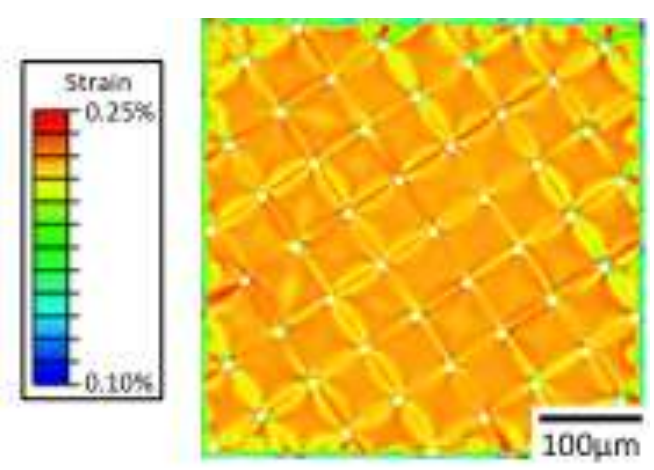

Figure 5. Strain distribution in a $22 \mathrm{~nm} \mathrm{Si} \mathrm{NM} \mathrm{substrate} \mathrm{after} 125 \mathrm{~nm} \mathrm{SiN}_{\mathrm{x}}$ film deposition as modeled with FEA. A uniform system strained was applied to an initially unstrained Si NM. The resulting strain distribution is a result of the constrained edges and the array of etchant access holes.

Figure 6 shows the NM deflections caused by the introduction of tensile strain into the Si NM and the constraint of the fixed edges. Figures 6A and B show the buckling of a 22nm Si NM before and after deposition of a $125 \mathrm{~nm} \mathrm{SiN}_{\mathrm{x}}$ film, respectively (corresponding to the parameters also shown in Fig. 5), as measured with a white-light interferometer. The Si NM substrate is slightly buckled before deposition because some strain is relaxed after removing the photoresist during sample preparation (Fig. 2D). Figures 6C and D show the buckling results of FEA of a similar bilayer structure before and after film deposition, respectively. The initial buckling pattern was chosen to match the experimental results of the Si NM substrate after removal of the photoresist (Figure 6A). The magnitude of the deflection after $\mathrm{SiN}_{\mathrm{x}}$ deposition is similar to that of the measured deflection. The buckling pattern of the FEA model does not appear to replicate the experimental result. Experimentally we observe several different buckling patterns for similar tethered Si NM substrates, and thus this outcome was not unexpected. We verified that there 
were multiple low-energy buckling configurations in the model as well, by changing the FE mesh size. The magnitude of the maximum deflection in the Si NM, however, closely matches the experimental results in all cases.
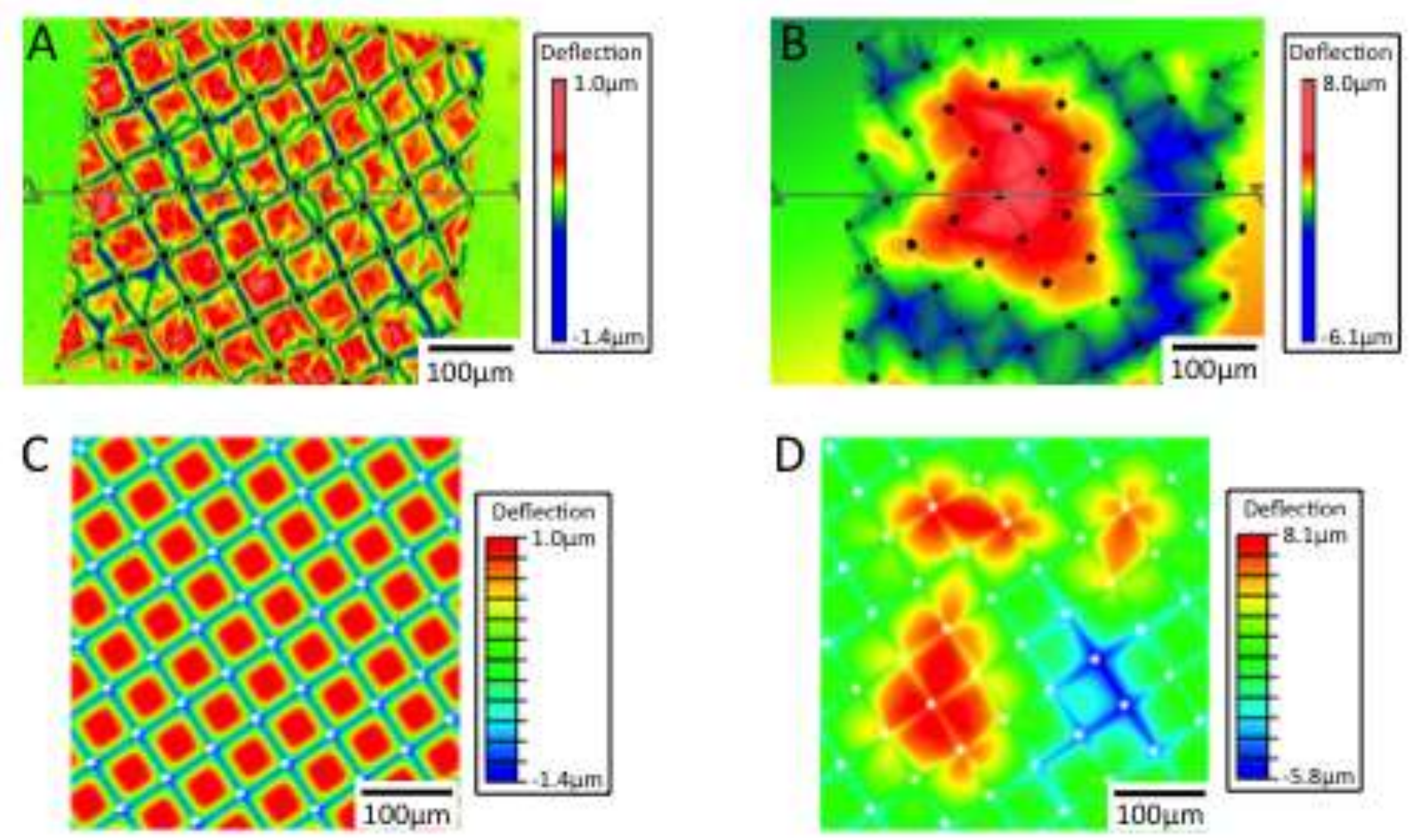

Figure 6. Deflection of a 22nm tethered Si NM before (A and C, experiment and FEA respectively) and after (B and D, experiment and FEA respectively) $125 \mathrm{~nm} \mathrm{SiN}{ }_{x}$ deposition. The experimental initial (A) and final (B) deflections are measured with white-light interferometry. Different buckling configurations after film deposition can be obtained both in experiment and in FEA modeling.

\section{Conclusions}

We used tethered Si NM substrates with thicknesses between 9 and 200nm to lay the basis for understanding stress evolution in films deposited on ultrathin compliant substrates. We deposited compressively stressed amorphous $\mathrm{SiN}_{\mathrm{x}}$. We independently varied the thickness of the $\mathrm{SiN}_{\mathrm{x}}$ film and the thickness of the tethered Si NM substrate. The strain in the Si NM substrate as a function of film thickness and substrate thickness suggests that the steady-state stress of the 
deposited film decreases with decreasing compliant-substrate thickness. The reduced steady-state film stress and resulting increased substrate strain are a result of the dynamic response of the substrate during film deposition; the substrate responds to the stress in the film as it is being deposited (in our case expands). The result is an ever-changing system strain. The final steady-state stress in the film is determined by how much the substrate expands or contracts during deposition and is thus dependent on the thickness of the substrate.

We modeled the strain distribution and deformation of the Si NM substrate after $\operatorname{SiN}_{\mathrm{x}}$ deposition with FEA. The maximum deflection and average strain match the experimental results. The strain distribution is uniform away from the tethered edges of the substrate and far from the etchant access holes: areas where the strain measurements were taken. The FEA model confirms that the direction and magnitude of the Si NM buckling does not significantly change the macroscopic strain distribution or the average strain.

These experiments represent only the first step in creating probes that could dynamically measure film stress evolution during growth on ultrathin substrates, and thus to understand better the mesoscopic mechanisms involved in such growth.

\section{Methods}

\subsection{Si NM window fabrication}

The template layer of $\mathrm{SOI}(001)$ is patterned with photolithography and reactive ion plasma etching to define the shape and size of the NM (usually $2-3 \mathrm{~mm}$ square) and any etchant access holes $(\sim 10-30 \mu \mathrm{m}$ on a side spaced $60-100 \mu \mathrm{m}$ apart $)$ [Figure $2 \mathrm{~A}]$. The Si template layer is separated from the bulk $\mathrm{Si}$ handle wafer by removing the $\mathrm{SiO}_{2}$ layer of the SOI via chemical etching in hydrofluoric acid (HF) [Figure 2B]. The released Si NM floats in water and is transferred over a square hole (smaller in area than the Si NM, usually $\sim 500 \times 500 \mu \mathrm{m}^{2}$ ) in a new Si wa- 
fer [Figure 2C]. The transferred Si NM is left to sit overnight to allow excess liquid to evaporate and to initiate bonding of the Si NM to the Si wafer. The photoresist from the photolithography step is kept on the Si NM during transfer, but is removed with $\mathrm{O}_{2}$ plasma etching after the Si NM is bonded to the Si wafer (Figure 2D).

\subsection{SiNx deposition and properties}

The $\mathrm{SiN}_{\mathrm{x}}$ is formed from $\mathrm{SiH}_{4}, \mathrm{NH}_{3}$, and $\mathrm{N}_{2}$ precursors at a substrate temperature of $250^{\circ} \mathrm{C}$. Varying the chamber pressure, plasma power and frequency, the deposition temperature, and precursor gas ratios will change the stress in the $\operatorname{SiN}_{x}$ film $[29,30]$.

Nanoindentation using a Hysitron (Minneapolis, MN, USA) TriboIndenter® equipped with a Berkovich probe was used to assess the Young's modulus, $E$, of $\mathrm{SiN}_{\mathrm{x}}$ films deposited on bulk $\mathrm{Si}(001)$ wafers ranging in thickness from $60-182 \mathrm{~nm}$. A series of 100 load-controlled nanoindents with $2 \mathrm{~s}$ of load, $4 \mathrm{~s}$ of hold at a maximum load, and $2 \mathrm{~s}$ of unload, with maximum loads ranging from 0.3 to $10 \mathrm{mN}$, were placed on each film. A 20nm lift-off and approach was also performed at the beginning of each nanoindent to define the zero value of load and depth. Additionally, a Quesant (Agoura Hills, CA, USA) atomic force microscope (AFM) incorporated into the TriboIndenter® enclosure was used to make $4 \mu \mathrm{m}$ field-of-view-images of 10 nanoindents in each series. The influence of the substrate on the modulus, $\mathrm{E}$, of the $\mathrm{SiN}_{\mathrm{x}}$ film are accounted for by comparing the measured effective Young's modulus, $E_{\text {eff }}$, to theoretical simulations [31], following previous work [32].

\subsection{Raman spectroscopy}

We measure the strain in the Si NM substrates with micro-Raman microscopy (LabRAM Aramis Horiba Jobin Yvon Confocal Raman Microscope). We use a laser wavelength of 442nm (HeCd laser), filtered to $1 \mathrm{~mW}$ to avoid excessive heating of the thin $\mathrm{Si} \mathrm{NM}$ substrate. The $\mathrm{SiN}_{\mathrm{x}}$ film is 
transparent to the $442 \mathrm{~nm}$ light; the penetration depth in $\mathrm{Si}$ is $~ 300 \mathrm{~nm}$ [5]. The Si NM substrates are much thinner than the penetration depth of the laser, which means that the resulting measurement is an average through the thickness of the $\mathrm{Si}$. The laser is focused with a 100x objective lens to a $\sim 700 \mathrm{~nm}$ spot size. The frequency of the Raman active mode from the freestanding portion of the Si NM substrate shifts linearly as a function of biaxial strain [6]. The frequency shifts are extracted by peak fitting and using the unstrained bulk-Si peak as calibration. Reference bulk-Si Raman spectra are always measured between the strained-Si NM spectra to eliminate the influence of possible instrument drift. A Voigt function is used to fit the peaks.

\section{Acknowledgements}

The authors would like to thank George Celler for providing the substrate material and the USDA Forest Products Laboratory for the use of their nanoindenter. We thank Thomas Wermelinger, Ralph Spolenak, and Shelley Scott for extensive discussions and for motivating this work. This research was supported by DOE, Grant No. DE-FG02-03ER46028. Facilities support (interferometer, Raman spectrometer) by the NSF MRSEC program is acknowledged. D.M.P. acknowledges support from the NSF Graduate Research Fellowship Program.

\section{Conflict of Interest}

The authors declare they have no conflicts of interest. 


\section{$\underline{\text { References }}$}

[1] G. Stoney, The Tension of Metallic Films Deposited by Electrolysis, Proc. Royal Soc. A82 (1909) 172.

[2] L. B. Freund, S. Suresh, Thin Film Materials: Stress Defect Formation, and Surface Evolution, Cambridge Univ. Press: Cambridge (2003).

[3] M. Huang, P. Rugheimer, M. G. Lagally, F. Liu, Bending of Nanoscale Ultrathin Substrates by Growth of Strained Thin Films and Islands, Phys. Rev. B 72 (2005) 085450.

[4] T. Wermelinger, C. Charpentier, M. D. Yuksek, R. Spolenak, Measuring Stresses in Thin Metal Films by Means of Raman Microscopy Using Silicon as a Strain Gage Material, J. Raman Spectrosc. 40 (2009) 1849.

[5] I. DeWolf, Micro-Raman Spectroscopy to Study Local Mechanical Stress in Silicon Integrated Circuits, Semicond. Sci. Technol. 11 (1996) 139-154.

[6] S. Nakashima, T. Mitani, M. Ninomiya, K. Matsumoto, Raman Investigation of Strain in Si/SiGe Heterostructures: Precise Determination of the Strain-Shift Coefficient of Si bands, J. Appl. Phys. 99 (2006) 053512.

[7] J. A. Rogers, M. G. Lagally, R. G. Nuzzo, Synthesis, Assembly and Applications of Semiconductor Nanomembranes, Nature 477 (2011) 45.

[8] S. A. Scott, M. G. Lagally, Elastically Strain-Sharing Nanomembranes: Flexible and Transferable Strained Silicon and Silicon-Germanium Alloys, J. Phys. D: Appl. Phys. 50 (2007) R75.

[9] F. Cavallo, M. G. Lagally, Semiconductors Turn Soft: Inorganic Nanomembranes, Soft Matter 6 (2010) 439. 
[10] M. Huang, F. Cavallo, F. Liu, M. G. Lagally, Nanomechanical Architecture of Semiconductor Nanomembranes, Nanoscale 3 (2011) 96.

[11] C. Euaruksakul, F. Chen, B. Tanto, C. S. Ritz, D. M. Paskiewicz, F. J. Himpsel, D. E. Savage, Y. Yao, F. Liu, M. G. Lagally, Relationships Between Strain and Band Structure in Si(001) and Si(110) Nanomembranes, Phys. Rev. B 80 (2009) 115323.

[12] J. R. Sánchez-Pérez, C. Boztug, F. Chen, F.F. Sudradjat, D. M. Paskiewicz, RB Jacobson, M. G. Lagally, R. Paiella, Direct-Bandgap Light-Emitting Germanium in Tensilely Strained Nanomembranes, P. Natl. Acad. Sci. 108 (2001) 18893.

[13] D. M. Paskiewicz, S. A. Scott, D. E. Savage, G. K. Celler, M. G. Lagally, Symmetry in Strain Engineering of Nanomembranes: Making New Strained Materials, ACS Nano 5 (2011) 5532.

[14] M. M. Roberts, L. J. Klein, D. E. Savage, K. A. Slinker, M. Friesen, G. Celler, M. A. Eriksson, M G. Lagally, Elastically Relaxed Free-Standing Strained-Silicon Nanomembranes, Nature Mater. 5 (2006) 388.

[15] D. M. Paskiewicz, D. E. Savage, M. V. Holt, P. G. Evans, M. G. Lagally, NanomembraneBased Materials for Group IV Semiconductor Quantum Electronics, Sci. Rep. 4 (2014) 4218.

[16] C. Deneke, R. Songmuang, N. Y. Jin-Phillipp, O. G. Schmidt, The Structure of Hybrid Radial Superlattices, J. Phys. D: Appl. Phys. 42 (2009) 103001.

[17] M. Yu, Y. Huang, J. Ballweg, H. Shin, M. Huang, D. E. Savage, M. G. Lagally, E. W. Dent, R. H. Blick, J. C. Williams, Semiconductor Nanomembrane Tubes: Three Dimensional Confinement for Controlled Neurite Outgrowth, ACS Nano 5 (2011) 2447. 
[18] W. Huang, S. Koric, X. Yu, K. J. Hsia, X. Li, Precision Structural Engineering of SelfRolled-Up 3D Nanomembranes Guided by Transient Quasi-Static FEM Modeling, Nano Lett. 14 (2014) 6293.

[19] F. Cavallo, K. T. Turner, M. G. Lagally, Facile Fabrication of Ordered CrystallineSemiconductor Microstructures on Compliant Substrates, Adv. Funct. Mater. 24 (2014) 1730.

[20] M. Ying, A. P. Bonifas, N. Lu, Y. Su, R. Li, H. Cheng, A. Ameen, Y. Huang, J. A. Rogers, Silicon Nanomembranes for Fingertip Electronics, Nanotechnol. 23 (2012) 344004.

[21] J.-H. Ahn, H.-S. Kim, K. J. Lee, Z. Zhu, E. Menard, R. G. Nuzzo, J. A. Rogers, HighSpeed Mechanically Flexible Single-Crystal Silicon Thin-Film Transistors on Plastic Substrates, IEEE Electr. Device L. 27 (2006) 460.

[22] H.-C. Yuan, J. Shin, G. Qin, L. Sun, P. Bhattacharya, M. G. Lagally, G. K. Celler, Z. Ma, Flexible Photodetectors on Plastic Substrates by Use of Printing Transferred SingleCrystalline Germanium Membranes, Appl. Phys. Lett. 94 (2009) 013102.

[23] H. Zhou, J.-H. Seo, D. M. Paskiewicz, Y. Zhu, G. K. Celler, P. M. Voyles, W. Zhou, M. G. Lagally, and Z. Ma, Fast Flexible Electronics with Strained Silicon Nanomembranes, Sci. Rep. 3 (2013) 1291.

[24] G. Qin, J.-H. Seo, Y. Zhang, W. Zhou, Y. Wang, J. Ma, Z. Ma, RF Characterization of Gigahertz Flexible Silicon Thin-Film Transistor on Plastic Substrates Under Bending Conditions, IEEE Electr. Device L. 34 (2013) 262.

[25] H. C. Ko, M. P. Stoykovich, J. Song, V. Malyarchuk, W. M. Choi, C.-J. Yu, J. B. Geddes III, J. Xiao, S. Wang, Y. Huang, J. A. Rogers, A Hemispherical Electronic Eye Camera Based on Compressible Silicon Optoelectronics, Nature 454 (2008) 748. 
[26] F. Liu, M. Huang, P. P. Rugheimer, D. E. Savage, M. G. Lagally, Nanostressors and the Nanomechanical Response of a Thin Silicon Film on an Insulator, Phys. Rev. Lett. 89 (2002) 136101.

[27] C. Deneke, A. Malachias, A. Rastelli, L. Merces, M. Huang, F. Cavallo, O. G. Schmidt, M. G. Lagally, Straining Nanomembranes via Highly Mismatched Heteroepitaxial Growth: InAs Islands on Compliant Si Substrates, ACS Nano 6 (2012) 10287.

[28] C. S. Ritz, H.-J. Kim-Lee, D. M. Detert, M. M. Kelly, F. S. Flack, D. E. Savage, Z. Cai, P. G. Evans, K. T. Turner, M. G. Lagally, Ordering of Nanostressors on Free-Standing Silicon Nanomembranes and Nanoribbons, New J. Phys. 12 (2010) 103011.

[29] Y. Toivola, J. Thurn, R. F. Cook, G. Cibuzar, K. Roberts, Influence of Deposition Conditions on Mechanical Properties of Low-Pressure Chemical Vapor Deposited Low-Stress Silicon Nitride Films, J. Appl. Phys. 94 (2003) 6915.

[30] E. Johlin, N. Tabet, S. Castro-Galnares, A. Abdallah, M. I. Bertoni, T. Asafa, J. C. Grossman, S. Said, T. Buonassisi, Structural Origins of Intrinsic Stress in Amorphous Silicon Thin Films, Phys. Rev. B. 85 (2012) 075202.

[31] D. S. Stone, Elastic Rebound Between an Indenter and a Layered Specimen. Part I. Model, J. Mat. Res. 13 (1998) 3207.

[32] J. E. Jakes, C. R. Frihart, J. F. Beecher, R. J. Moon, P. J. Resto, Z. H. Melgarejo, O. M. Surez, H. Baumgart, A. A. Elmustafa, and D. S. Stone, Nanoindentation Near the Edge, J. Mat. Res. 24 (2009) 1016. 